\title{
Patterns of caesarean section in HIV infected and non-infected women in Malawi: is caesarean section used for PMTCT?
}

Lyson Tenthani ${ }^{1,2,3^{*}}$, Joep J. van Oosterhout ${ }^{4,5}$, Andreas D. Haas ${ }^{2}$, Malango Msukwa ${ }^{6,8}$, Nozgechi Phiri ${ }^{6,8}$, Frank Chimbwandira', Kali Tal' ${ }^{2}$, Karoline Aebi-Popp ${ }^{7}$, Janne Estill ${ }^{2,8,9}$ and Olivia Keiser ${ }^{2,8}$

\begin{abstract}
Background: Caesarean section (CS) is not recommended for PMTCT in Malawi HIV Guidelines, contrary to most high-income countries where CS is indicated if viral suppression is sub-optimal pre-delivery. We describe patterns of CS in HIV-infected and uninfected women in Malawi and explored if insight into the use of Elective CS (ECS) for PMTCT could be obtained.

Methods: We used routinely collected data from individual medical records from 17 large health facilities in the central and southern regions of Malawi, from January 2010 to December 2013. We included data from maternity registers from all HIV-positive women, and randomly selected around every fourth woman with negative or unknown HIV status. We used multivariable logistic regressions and cluster-based robust standard errors to examine independent associations of patient- and facility characteristics with CS and ECS.

Results: We included 62,033 women in the analysis. The weighted percentage of women who had a spontaneous vaginal delivery was $80.0 \%$ (Cl 95\% 79.5-80.4\%); $2.4 \%$ (95\% Cl 2.3-2.6\%) had a vacuum extraction; $2.3 \%$ (95\% Cl 2.22.5\%) had a vaginal breech delivery; $14.0 \%(95 \% \mathrm{Cl} 13.6-14.4 \%)$ had a CS while for $1.3 \%(95 \% \mathrm{Cl} 1.2-1.4 \%)$ the mode of delivery was not recorded. Prevalence of CS without recorded medical or obstetric indication (ECS) was 5. $1 \%$, $(n=3152)$. Presence of maternal and infant complications and older age were independently associated with CS delivery. HIV-positive women were less likely to have ECS than HIV negative women (aOR 0.65; 95\%-CI 0.57-0. 74). Among HIV-positive women, those on antiretrovirals (ARV's) for $\geq 4$ weeks prior to delivery were less likely to have ECS than HIV-positive women who had not received ARVs during pregnancy (aOR 0.81; 95\% Cl 0.68-0.96).

Conclusions: The pattern of CS's in Malawi is largely determined by maternal and infant complications. Positive HIV status was negatively associated with CS delivery, possibly because health care workers were concerned about the risk of occupational HIV transmission and the known increased risk of post-operative complications. Our results leave open the possibility that CS is practiced to prevent MTCT given that ECS was more common among women at high risk of MTCT due to no or short exposure to ARV's.
\end{abstract}

\footnotetext{
* Correspondence: Itenthani@gmail.com

${ }^{1}$ Department of HIV and AIDS, Ministry of Health, Lilongwe, Malawi

${ }^{2}$ Institute of Social and Preventive Medicine, University of Bern, Bern, Switzerland

Full list of author information is available at the end of the article
} 


\section{Background}

In 2014, an estimated 1.1 million people were living with HIV in Malawi, including 130,000 children under fifteen [1]. Mother-to-child transmission (MTCT) is the most common cause of infection in children. Without intervention, risk of MTCT is estimated to be $5-10 \%$ over the course of pregnancy, 10-20\% during labour, and 10$20 \%$ during breastfeeding; overall $30-45 \%$ of infants born to mothers with HIV will contract the virus [2]. With effective antiretroviral therapy (ART), the risk of MTCT can be reduced to less than 5\% [3].

In Malawi the total fertility rate is estimated to be between 4.4 and 5.7 children per woman. High ratios of maternal mortality (460-680 per 100,000 live births) and neonatal mortality (27 per 1000 live births) have been recorded. The contraceptive prevalence rate varies between $44.4 \%$ (sexually active unmarried women) and 59. 2\% (married women) [4]. Maternal mortality is believed to be strongly impacted on by the HIV epidemic, with a steep increase between 1992 and 2000 and a sharp decline since the start of free ART provision in 2004 [4].

In this context, Malawi implemented a test and treat policy ("Option $\mathrm{B}+$ ") to facilitate access to ART for all HIV-infected pregnant and breastfeeding women [4]. The policy was adopted by other low-income countries and is now recommended by the World Health Organization [5]. Under Option $\mathrm{B}+$, women are diagnosed with HIV during routine testing in antenatal care and they initiate ART within one week of diagnosis [4]. In 2011, Option B+ was implemented as Malawi's National PMTCT policy and the ART coverage among pregnant women increased strongly [6]. However, even with Option B+, a substantial proportion of women are likely not to be fully virologically suppressed when around $20 \%$ of pregnant women are not tested for HIV [7]; almost 20\% discontinue treatment within the first months [8, 9]; and about $30 \%$ adhere to ART inadequately during pregnancy, with young age and Option $\mathrm{B}+$ indication for ART as risk factors [10]. Overall, 9\% of women who are on ART during pregnancy do not achieve adequate virological suppression at delivery [11].

Caesarean section (CS) can be used to reduce the risk of intrapartum HIV transmission [12-14]. In high-income countries CS is recommended for women with $>400 \mathrm{HIV}$ RNA copies/ml at the time of delivery $[15,16]$. In resource constrained settings like Malawi, elective caesarean section (ECS; defined here as a CS without documented obstetric or medical indication) is not a recommended PMTCT strategy [17] as the increased risk of morbidity and mortality that is associated with ECS is expected to outweigh its HIV prevention benefits $[18,19]$. In addition, resources and capacity to perform CS are limited in lowincome countries like Malawi [19, 20]. Despite this, it is possible that health care workers and pregnant women in
Malawi opt for an ECS in circumstances where MTCT risk is known or believed to be high.

We describe the prevalence and pattern of indications of CS among deliveries of HIV positive and HIV negative women in Malawi. We also explore our data for the potential practice of using ECS for PMTCT.

\section{Methods}

Data sources

We entered routinely collected data from individual medical records in 17 large health facilities in the central and southern regions of Malawi, from January 2010 to December 2013. The selected facilities were among 20 study facilities that participate in the Umoyo+ study (http:// aidsinfo.unaids.org/). Two of the 20 facilities were excluded because no CS's were done, another one because of missing data. In the Malawi government health care system, observations from labour and delivery are recorded on labour charts and summarized in a maternity register. These standard monitoring and evaluation tools capture demographic characteristics, obstetrical history, and observations on the delivery and infant. Theatre registers document detailed information about CS (i.e. indication, duration, observations and outcome of the procedure). At each health facility, we included data from maternity registers from all HIV positive women and from a randomly selected approximately one quarter of women with negative or unknown HIV status. We describe the sampling strategy in more detail in the Additional file 1: Appendix 1. In case the indication for CS could not be determined from the maternity register, we extracted relevant information from labour charts and theatre registers.

\section{Definitions}

Health care workers classified the mode of delivery either as $\mathrm{CS}$ or as vaginal delivery (including assisted vaginal deliveries that use vacuum and forceps extraction). The main outcomes of the study were CS and ECS. The delivery was defined as CS when it was conducted through CS irrespective of the reason for the procedure. A CS was classified as ECS if it was conducted electively without documentation of an obstetric or medical indication. In women with a previous CS, the current CS was counted as ECS only if there was no trial of vaginal delivery for the current delivery with no previous history of CS (if a previous CS was the indication for the current CS, this will be reported according to local guidelines). HIV status was positive if a woman was admitted for labour and delivery with documentation of a positive HIV test result or if she was on ART, or if she had a positive test result obtained between the onset of labour and the period immediately after delivery. A woman was classified as HIV negative if she had a negative test result in that same period or if she had a documented negative test result during the current pregnancy. HIV unknown status was assigned if 
testing was not documented, if it was documented as not done, or if test results were inconclusive or missing.

\section{Statistical analysis}

We provide patterns of indications for CS among HIV positive, unknown and negative women using descriptive statistics. To examine independent associations between CS or ECS and patient and facility characteristics, we used multivariable logistic regression analyses, with cluster-based robust standard errors adjusting for clustering of patients within facilities. We considered the following explanatory variables: year of admission, maternal complications (hemorrhage, obstructed/prolonged labour, pre-eclampsia, maternal sepsis, ruptured uterus, others when unspecified, unknown if nothing was recorded), infant complications (prematurity, low birth weight, asphyxia, other if unspecified, unknown if nothing was recorded), HIV status (negative, positive, unknown), and ARV use during pregnancy (no ARVs, ARVs for $<4$ weeks, ARVs for $\geq 4$ weeks), number of deliveries $(0,1,2-3,>3)$, singleton (yes/no), age category (<20, 20-24, 25-29, 30-34, > 34 years), facility ownership (government or Christian Health Association (CHAM)) and location (urban or rural). We ran 4 models each to examine predictors of CS and predictors of ECS. We included all women in the first CS model; women with unknown HIV status in the second, HIV negative women in the third model and HIV positive women in the fourth. In the four ECS models, we excluded women who had a previous CS and failed trial of vaginal delivery and women with a recorded maternal or infant complication (as these women were not at risk of ECS) (Fig. 1). In all analyses HIV negative women were weighted by the inverse of the probability that the observation was included because of the sampling design. HIV positive women were assigned a weight of 1 as data from all HIV positive women was analyzed (see sampling strategy description in Additional file 1: Appendix 1). We therefore report weighted prevalence rates throughout. All data were analyzed with STATA software (Version 13.1, Stata Corporation, Texas USA.

\section{Results}

\section{Characteristics of study participants}

Out of 62,033 women included in the analysis 56,292 (90. 7\%) had a known HIV status and 5741 (9.3\%) had an unknown HIV status. Among women with known HIV status 23,193 were HIV positive and 33,099 were HIV negative, resulting in a weighted HIV prevalence of $9.7 \%$. (95\% CI 9. 3-10.1\%). Characteristics of study participants stratified

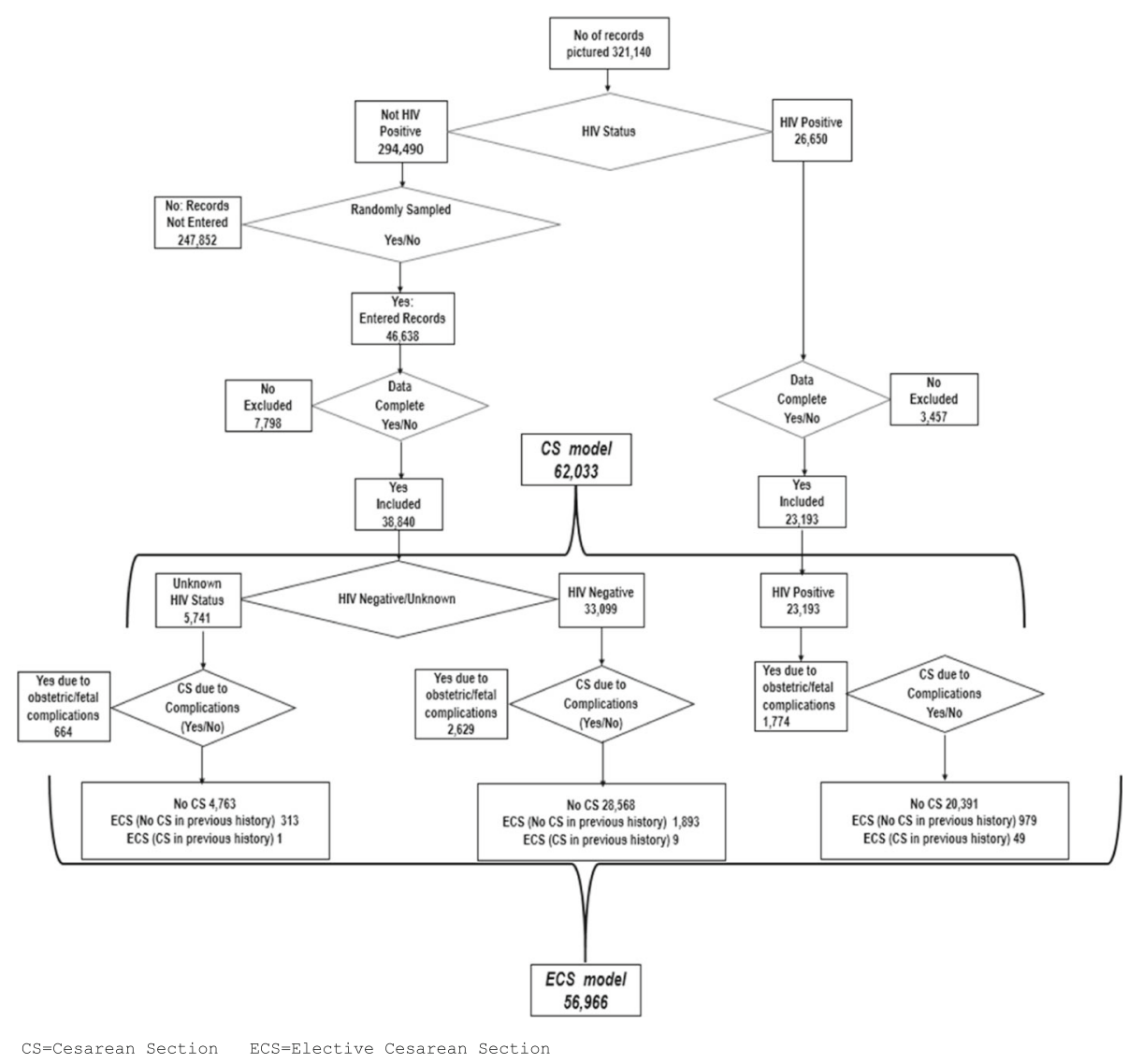

Fig. 1 Flow chart showing the patients' records included in the study and the specific model according to their HIV status and mode of delivery 
by HIV status are shown in Table 1 . Their median age was 25 years (interquartile range [IQR] 20-30). Women had a median of one delivery (IQR 1-3). The majority of women $(89.6 \%, 55,583)$ received care at government owned facilities and 10.4\% (6450) were managed in faith-based clinics. About $76 \%$ of the women $(47,215)$ attended clinics in rural settings and $24 \%(14,818)$ were seen at urban clinics.

\section{Maternal and infant complications}

The proportion of women with maternal complications was $12.5 \%(95 \%$ CI 12.1-12.9\%). Maternal complications occurred more frequently among HIV positive (15.2\%; 95\% CI 14.8-15.7\%) than among HIV negative women (11.5\%; 95\% CI 11.1-11.9\%). Among women with unknown HIV status the corresponding percentage was 16.2\% (95\% CI 15. $2-17.3 \%)$. The percentage of infants who had complications was $14.5 \%$ (95\% CI 14.1-14.8\%). Infant complications were more common among HIV exposed infants (25.1\%, CI 24. 5-25.7\%) than among infants born to mothers with negative $(12.7 \%$, CI $12.3-13.1 \%)$ or unknown HIV status (18.3\%, CI 17.2-19.5\%). The distribution of specific maternal and infant complications by HIV status is shown in Table 1.

\section{Mode of delivery}

The percentage of women who had a spontaneous vaginal delivery was $80.0 \%$ (CI 95\% 79.5-80.4\%); $2.4 \%$ (95\% CI $2.3-2.6 \%$ ) had vacuum extraction; $2.3 \%$ (95\% CI $2.2-$ $2.5 \%$ ) had a vaginal breech delivery; $14.0 \%$ (95\% CI 13.6$14.4 \%$ ) had CS while for $1.3 \%$ (95\% CI 1.2-1.4\%) of the women mode of delivery was not recorded.

Overall, prevalence of CS among women with unknown HIV status was significantly higher than among deliveries of HIV negative women, which in turn was significantly higher than among deliveries in HIV positive women (HIV unknown 16.2\%, 95\% CI 15.1-17.3\%; HIV negative 13.8\%, 95\% CI 13.3-14.2\%; HIV positive $12.1 \%$, 95\% CI 11.7-12.5\%). The distribution of CS according to individual and facility-level characteristics stratified by HIV status is shown in Table 2. Among women with a recorded complication 64.4\% (95\% CI 63.9-64.9\%) had a CS delivery compared to $6.8 \%$ (95\% CI 6.7-6.9\%) in women without a recorded complication. The prevalence of elective CS without recorded medical or obstetric indication (ECS) was $5.1 \%$ (95\% CI $4.9-5.3 \%)$.

\section{Associations of individual and facility level characteristics with CS}

HIV positive women were less likely to have CS compared to HIV negative women (aOR 0.60; 95\%-CI 0.51-0.71) while those with unknown HIV status had similar odds of CS as HIV negative women (aOR 1.01; 96\% CI 0.84-1.20). Table 3 shows the results from the multivariable logistic regression analyses of variables associated with CS. Presence of maternal complications, infant complications and older age were independently associated with CS delivery in each of the three groups. Twin delivery and delivering for the first time were associated with a higher probability of CS in all groups. Year of admission was independently associated with a lower odds of CS after 2010 only in HIV negative women.

\section{Associations of individual and facility level characteristics with ECS}

Results from the multivariable logistic regression analyses of variables associated with ECS delivery among all women, HIV positive women, those with unknown HIV status and HIV negative women are presented in Table 4. HIV positive women were less likely to have ECS compared to HIV negative women (aOR 0.65; 95\%-CI 0.57-0.74). Among HIV positive women, those who were on ARV's for $\geq 4$ weeks prior to delivery were less likely to have ECS than HIV positive women who had not received ARVs during pregnancy. Older age and delivery in an urban facility were associated with higher odds of ECS in all groups. First deliveries and non-singleton deliveries were independently associated with an increased odds of ECS in all groups except in women with HIV unknown status. Delivery in 2010, was independently associated with ECS, except in HIV positive women.

\section{Discussion}

We found that the prevalence of CS in deliveries in 17 large health facilities in Malawi was $14.0 \%$ and was higher among deliveries of HIV negative women compared to HIV positive women. The $14 \%$ CS prevalence found in our study is almost three times higher than the $5 \%$ last reported in the 2010 nationally representative study $[21,22]$ and the $6.2 \%$ reported in a multinational study in sub-Saharan Africa [23]. However this can be explained by our exclusion of deliveries taking place in primary health facilities where CS services are not offered.

CS delivery was strongly associated with presence of maternal or infant complications. As expected, maternal and infant complications were more common among HIV positive women than HIV negative women, while those with unknown HIV status had similar frequency of complications to HIV positive women (possibly due to undiagnosed HIV among those with unknown status). We also found that among HIV positive women the chance of ECS was higher in those who had received less than 4 weeks or no exposure to ARVs during pregnancy. As in other studies the adjusted odds of a CS delivery increased with age and almost doubled among HIV positive women aged above 29 years compared to those below 20 years [24-27].

Our data suggest that patterns of CS in Malawi are also influenced by other factors than maternal and fetal obstetric conditions. The lower frequency of CS among HIV positive women in spite of their higher maternal and infant complication rates could be explained by 
Table 1 Characteristics of study participants by HIV status

\begin{tabular}{|c|c|c|c|c|c|c|c|c|c|}
\hline \multirow[t]{2}{*}{ Item } & & \multicolumn{2}{|c|}{$\begin{array}{l}\text { All women } \\
(n=62,033)\end{array}$} & \multicolumn{2}{|c|}{$\begin{array}{l}\text { Women with unknown } \\
\text { HIV status }(n=5741)\end{array}$} & \multicolumn{2}{|c|}{$\begin{array}{l}\text { HIV negative women } \\
(n=33,099)\end{array}$} & \multicolumn{2}{|c|}{$\begin{array}{l}\text { HIV positive women } \\
(n=23,193)\end{array}$} \\
\hline & & Total & Weighted \% & Total & Weighted \% & Total & Weighted \% & Total & Weighted \% \\
\hline \multirow[t]{4}{*}{ Year of admission } & 2010 & 10,526 & 20.8 & 675 & 18.4 & 4272 & 20.9 & 5579 & 24.1 \\
\hline & 2011 & 16,216 & 30.9 & 1647 & 38.2 & 7056 & 29.4 & 7513 & 32.4 \\
\hline & ‘2012 & 16,749 & 22.6 & 1888 & 23.6 & 9889 & 22.5 & 4972 & 21.4 \\
\hline & 2013 & 18,542 & 25.8 & 1531 & 19.9 & 11,882 & 27.2 & 5129 & 22.1 \\
\hline \multirow[t]{8}{*}{ Maternal complication } & None & 52,992 & 86.8 & 4674 & 82.4 & 28,932 & 88.0 & 19,386 & 83.6 \\
\hline & Hemorrhage & 1566 & 2.0 & 173 & 2.9 & 621 & 1.7 & 772 & 3.3 \\
\hline & OPL & 2899 & 4.3 & 312 & 5.0 & 1413 & 4.7 & 1175 & 5.1 \\
\hline & Preeclampsia & 511 & 0.8 & 78 & 1.2 & 260 & 0.7 & 173 & 0.7 \\
\hline & Sepsis & 101 & 0.1 & 13 & 0.3 & 21 & 0.1 & 67 & 0.3 \\
\hline & $\mathrm{RU}$ & 161 & 0.2 & 28 & 0.5 & 68 & 0.2 & 65 & 0.3 \\
\hline & Unspecified & 3275 & 5.0 & 386 & 6.4 & 1610 & 4.7 & 1278 & 5.5 \\
\hline & Unknown & 528 & 0.7 & 77 & 1.4 & 174 & 0.5 & 277 & 1.2 \\
\hline \multirow[t]{6}{*}{ Infant complication } & None & 51,017 & 85.8 & 4691 & 82.1 & 28,892 & 87.6 & 17,435 & 75.2 \\
\hline & Prematurity & 2609 & 3.6 & 281 & 4.7 & 1163 & 3.3 & 1156 & 5.0 \\
\hline & LBW & 2166 & 3.0 & 261 & 4.8 & 844 & 2.5 & 1073 & 4.6 \\
\hline & Asphyxia & 2140 & 3.4 & 246 & 3.9 & 1158 & 3.3 & 732 & 3.2 \\
\hline & Unspecified & 1683 & 2.6 & 197 & 3.3 & 811 & 2.4 & 675 & 2.9 \\
\hline & Unknown & 2418 & 1.6 & 65 & 1.2 & 231 & 0.8 & 2122 & 9.1 \\
\hline \multirow[t]{5}{*}{ No of previous deliveries } & 0 & 14,446 & 29.1 & 1738 & 30.6 & 10,125 & 30.8 & 2583 & 11.1 \\
\hline & 1 & 12,554 & 21.2 & 1138 & 19.9 & 7314 & 21.8 & 4102 & 17.7 \\
\hline & $2-3$ & 19,780 & 28.0 & 1505 & 26.2 & 8856 & 26.9 & 9419 & 40.6 \\
\hline & $>3$ & 14,554 & 20.5 & 1180 & 20.4 & 6523 & 19.6 & 6851 & 29.5 \\
\hline & Unknown & 699 & 1.2 & 180 & 2.9 & 281 & 0.9 & 238 & 1.0 \\
\hline \multirow[t]{6}{*}{ Age in years } & $<20$ & 11,191 & 23.0 & 1428 & 26.3 & 8217 & 24.4 & 1546 & 6.7 \\
\hline & $20-24$ & 17,485 & 31.0 & 1738 & 30.5 & 10,491 & 32.0 & 5256 & 22.7 \\
\hline & $25-29$ & 14,003 & 20.0 & 1035 & 17.1 & 6374 & 19.5 & 6595 & 28.4 \\
\hline & $30-34$ & 10,960 & 13.9 & 740 & 12.5 & 4389 & 12.9 & 58,31 & 25.1 \\
\hline & $>34$ & 7288 & 10.2 & 583 & 9.8 & 3094 & 9.6 & 3610 & 15.6 \\
\hline & Unknown & 1106 & 1.9 & 217 & 4.0 & 534 & 1.6 & 355 & 1.5 \\
\hline \multirow[t]{2}{*}{ Singleton } & Yes & 59,862 & 96.6 & 5519 & 96.1 & 32,068 & 96.7 & 22,274 & 96.0 \\
\hline & No & 2171 & 3.4 & 222 & 3.9 & 1031 & 3.3 & 919 & 4.0 \\
\hline \multirow[t]{4}{*}{ ARVs during pregnancy } & None, HIV+ & 11,938 & 4.3 & \multicolumn{4}{|c|}{ N/A } & 11,938 & 51.5 \\
\hline & $<4 w k s$ & 2716 & 1.0 & & & & & 2716 & 11.7 \\
\hline & $\geq 4 \mathrm{wks}$ & 8539 & 3.1 & & & & & 8539 & 36.8 \\
\hline & None, HIV- & 38,840 & 91.6 & & & & & \multicolumn{2}{|r|}{ N/A } \\
\hline \multirow[t]{2}{*}{ Facility ownership } & Government & 55,583 & 89.6 & 5476 & 95.3 & 29,447 & 88.1 & 20,660 & 89.1 \\
\hline & CHAM & 6450 & 10.4 & 265 & 4.7 & 3652 & 11.9 & 2533 & 10.9 \\
\hline \multirow[t]{2}{*}{ Facility location } & Rural & 47,215 & 80.4 & 4936 & 86.2 & 26,660 & 80.8 & 15,619 & 67.3 \\
\hline & Urban & 14,818 & 19.6 & 805 & 13.8 & 6439 & 19.2 & 7574 & 32.7 \\
\hline
\end{tabular}


Table 2 Proportion of women who delivered through C-section according to baseline characteristics

\begin{tabular}{|c|c|c|c|c|c|}
\hline Item & & $\begin{array}{l}\text { All Women } \\
(n=62,033)\end{array}$ & $\begin{array}{l}\text { Women with unknown } \\
\text { HIV status }(n=5741)\end{array}$ & $\begin{array}{l}\text { HIV negative women } \\
\mathrm{n}=33,099)\end{array}$ & $\begin{array}{l}\text { HIV positive women } \\
\mathrm{n}=23,193\end{array}$ \\
\hline \multirow[t]{4}{*}{ Year of admission } & 2010 & $15.2(14.2-16.3)$ & $17.2(14.3-20.7)$ & $15.1(13.9-16.4)$ & $13.7(12.8-14.6)$ \\
\hline & 2011 & $13.7(12.9-14.5)$ & $13.4(11.7-15.4)$ & $13.8(12.9-14.9)$ & $13.3(12.5-14.1)$ \\
\hline & 2012 & $13.4(12.8-13.9)$ & $18.2(16.6-20.1)$ & $12.8(12.2-13.5)$ & $9.6(8.8-10.5)$ \\
\hline & 2013 & $13.8(13.3-14.4)$ & $18.1(16.2-20.1)$ & $13.5(12.9-14.1)$ & $11.0(10.2-11.9)$ \\
\hline \multirow[t]{8}{*}{ Maternal complication } & None & $6.6(6.3-6.9)$ & $6.3(6.1-6.6)$ & $6.8(6.6-6.9)$ & $5.1(4.8-5.4)$ \\
\hline & Hemorrhage & $29.5(26.5-32.8)$ & $34.7(32.0-37.6)$ & $30.2(28.7-31.7)$ & $19.0(16.4-22.0)$ \\
\hline & $\mathrm{OPL}$ & $68.2(65.8-70.4)$ & $68.0(65.9-70.0)$ & $69.2(68.2-70.1)$ & $60.9(58.0-63.6)$ \\
\hline & Preeclampsia & $60.2(55.0-65.2)$ & $64.8(60.4-69.1)$ & $61.2(58.7-63.6)$ & $39.3(32.3-46.8)$ \\
\hline & Sepsis & $16.5(7.3-33.3)$ & $5.1(2.1-11.5)$ & $30.7(23.4-39.2)$ & $6.0(2.2-14.9)$ \\
\hline & $R U$ & $87.4(78.5-93.0)$ & $84.7(78.6-89.4)$ & $90.7(87.6-93.1)$ & $72.3(60.2-81.9)$ \\
\hline & Unspecified & 75.7 (73.5-77.8) & 77.0 (75.3-78.6) & $77.2(76.3-78.0)$ & $61.4(58.7-64.1)$ \\
\hline & Unknown & $33.0(27.6-38.8)$ & $31.9(28.1-36.1)$ & $36.6(33.8-39.4)$ & $20.6(16.2-25.8)$ \\
\hline \multirow[t]{6}{*}{ Infant complication } & None & $12.4(12.0-12.8)$ & $13.9(13.5-14.3)$ & $12.3(12.1-12.4)$ & $11.2(10.7-11.7)$ \\
\hline & Prematurity & $17.0(15.1-19.1)$ & $21.7(19.9-23.7)$ & $16.1(15.3-17.0)$ & $15.2(13.3-17.4)$ \\
\hline & LBW & $20.8(18.4-23.4)$ & $16.1(14.5-17.8)$ & $23.0(21.9-24.1)$ & $18.0(15.8-20.4)$ \\
\hline & Asphyxia & $29.2(26.7-31.8)$ & $40.0(37.5-42.5)$ & $26.8(25.8-27.8)$ & $30.2(27.0-33.6)$ \\
\hline & Unspecified & $32.4(29.5-35.5)$ & $36.8(34.2-39.5)$ & $31.8(30.6-33.1)$ & $29.2(25.9-32.7)$ \\
\hline & Unknown & $15.7(12.9-19.1)$ & $15.5(12.5-19.0)$ & $31.2(29.1-33.4)$ & $2.7(2.1-3.5)$ \\
\hline \multirow[t]{5}{*}{ No of previous deliveries } & 0 & $16.3(15.5-17.1)$ & $16.2(15.5-16.9)$ & $16.2(16.0-16.5)$ & $18.7(17.3-20.3)$ \\
\hline & 1 & $14.2(13.3-15.0)$ & $15.9(15.1-16.8)$ & $14.0(13.7-14.3)$ & $12.6(11.6-13.6)$ \\
\hline & $2-3$ & $13.3(12.6-14.0)$ & $17.8(17.0-18.5)$ & $12.7(12.5-13.0)$ & $11.6(11.0-12.3)$ \\
\hline & $>3$ & $11.2(10.5-12.1)$ & $13.8(13.0-14.6)$ & $11.0(10.7-11.3)$ & $9.9(9.2-10.6)$ \\
\hline & Unknown & $17.4(14.1-21.3)$ & $20.2(18.0-22.7)$ & $16.2(14.6-17.9)$ & $14.3(10.4-19.3)$ \\
\hline \multirow[t]{6}{*}{ Age in years } & $<20$ & $14.3(13.5-15.1)$ & $13.3(12.6-14.0)$ & $14.5(14.2-14.8)$ & $13.6(12.0-15.5)$ \\
\hline & $20-24$ & $14.6(13.9-15.4)$ & $18.5(17.8-19.3)$ & $14.2(13.9-14.4)$ & $12.3(11.4-13.2)$ \\
\hline & $25-29$ & $14.6(13.8-15.5)$ & $18.7(17.8-19.6)$ & $14.4(14.1-14.7)$ & $11.8(11.1-12.6)$ \\
\hline & $30-34$ & $12.1(11.2-13.0)$ & $13.7(12.8-14.7)$ & $11.8(11.4-12.2)$ & $11.9(11.1-12.8)$ \\
\hline & $>34$ & $12.6(11.5-13.8)$ & $15.3(14.2-16.5)$ & $12.2(11.8-12.7)$ & $11.9(10.9-13.0)$ \\
\hline & Unknown & $13.6(11.1-16.4)$ & $15.3(13.6-17.3)$ & $13.0(11.9-14.1)$ & $12.4(9.3-16.3)$ \\
\hline \multirow[t]{2}{*}{ Singleton } & Yes & $13.7(13.4-14.1)$ & $16.0(15.7-16.4)$ & $13.6(13.4-13.7)$ & $11.7(11.3-12.2)$ \\
\hline & No & $20.4(17.9-23.0)$ & $19.7(17.8-21.8)$ & $20.4(19.5-21.4)$ & $20.8(18.2-23.4)$ \\
\hline \multirow[t]{4}{*}{ ARVs during pregnancy } & None & $11.7(11.1-12.3)$ & \multicolumn{2}{|c|}{ N/A } & $11.7(11.1-12.3)$ \\
\hline & $<4$ wks & $12.4(11.3-13.7)$ & & & $12.4(11.3-13.7)$ \\
\hline & $\geq 4 \mathrm{wks}$ & $12.5(11.9-13.2)$ & & & $12.5(11.9-13.3)$ \\
\hline & HIV- \& Unknown & $14.1(13.7-14.6)$ & & & N/A \\
\hline \multirow[t]{2}{*}{ Facility ownership } & Government & $13.8(13.4-14.2)$ & $16.2(15.8-16.5)$ & $13.5(13.4-13.7)$ & $12.2(11.8-12.7)$ \\
\hline & CHAM & $15.3(14.1-16.7)$ & $16.6(15.0-18.4)$ & $15.7(15.2-16.1)$ & $11.3(10.1-12.5)$ \\
\hline \multirow[t]{2}{*}{ Facility location } & Rural & $13.6(13.4-13.7)$ & $16.0(15.6-16.4)$ & $13.3(13.1-13.4)$ & $11.4(10.9-11.9)$ \\
\hline & Urban & $15.7(15.4-16.0)$ & $17.3(16.3-18.3)$ & $15.9(15.5-16.2)$ & $13.6(12.9-14.4)$ \\
\hline Totals & & $14.0(13.6-14.4)$ & $16.2(15.1-17.3)$ & $13.8(13.3-14.2)$ & $12.1(9.1-15.1)$ \\
\hline
\end{tabular}

OPL obstructed/prolonged labor, RU Ruptured Uterus, CHAM Christian Health Association of Malawi, LBW Low Birth Weight, aOR adjusted odds ratio, ARVs antiretrovirals 
Table 3 Individual and facility-level factors associated with C-Section among women delivering in 17 large health facilities in Malawi

\begin{tabular}{|c|c|c|c|c|c|c|c|c|c|}
\hline Item & & $\begin{array}{l}\text { aOR }(95 \% \mathrm{Cl}) \\
\text { All Women } \\
(55,443) \\
\end{array}$ & $p$ & $\begin{array}{l}\text { aOR }(95 \% \mathrm{Cl}) \text { Women } \\
\text { with Unknown HIV } \\
\text { status }(n=5302)\end{array}$ & $P$ & $\begin{array}{l}\text { aOR }(95 \% \mathrm{Cl}) \\
\text { HIV- Women } \\
(\mathrm{n}=31,978) \\
\end{array}$ & $p$ & $\begin{array}{l}\mathrm{aOR}(95 \% \mathrm{Cl}) \\
\mathrm{HIV}+\text { Women } \\
(n=20,436)\end{array}$ & $P$ \\
\hline \multirow{4}{*}{$\begin{array}{l}\text { Year of } \\
\text { admission }\end{array}$} & 2010 & 1 & 0.07 & 1 & 0.09 & 1 & 0.02 & 1 & 0.06 \\
\hline & 2011 & $0.71(0.50-1.01)$ & & $0.58(0.37-0.91)$ & & $0.71(0.50-1.00)$ & & $1.00(0.73-1.38)$ & \\
\hline & 2012 & $0.65(0.45-0.93)$ & & $0.71(0.42-1.19)$ & & $0.61(0.43-0.88)$ & & $0.83(0.56-1.22)$ & \\
\hline & 2013 & $0.67(0.49-0.92)$ & & $0.67(0.43-1.07)$ & & $0.65(0.48-0.87)$ & & $1.04(0.73-1.49)$ & \\
\hline \multirow{7}{*}{$\begin{array}{l}\text { Maternal } \\
\text { Obstetric } \\
\text { Complication }\end{array}$} & None & 1 & $<0.01$ & 1 & $<0.01$ & 1 & $<0.01$ & 1 & $<0.01$ \\
\hline & Hemorrhage & $5.85(3.12-10.97)$ & & $7.02(4.26-11.58)$ & & $5.80(3.03-11.12)$ & & $4.25(1.72-10.47)$ & \\
\hline & OPL & $32.23(17.15-60.57)$ & & $29.43(17.75-48.80)$ & & 33.61 (16.99-66.47) & & 29.88 (11.89-75.13) & \\
\hline & Preeclampsia & $21.76(10.32-45.86)$ & & $27.18(14.18-52.09)$ & & $22.14(9.83-49.88)$ & & $11.23(4.56-27.68)$ & \\
\hline & Sepsis & $4.25(1.30-13.85)$ & & $1.35(0.14-13.18)$ & & $8.13(1.57-42.16)$ & & $1.20(0.36-4.02)$ & \\
\hline & $\mathrm{RU}$ & $160.25(75.41-340.51)$ & & 141.95 (34.98-576.08) & & $186.48(66.45-523.27)$ & & 94.91 (43.33-207.89) & \\
\hline & Others & $52.85(31.25-89.39)$ & & $51.75(35.26-75.97)$ & & $55 .-81$ (33.24-93.69) & & 38.01 (15.13-95.52) & \\
\hline \multirow{5}{*}{$\begin{array}{l}\text { Infant } \\
\text { Complication }\end{array}$} & None & 1 & 0.01 & 1 & $<0.01$ & 1 & $<0.01$ & 1 & $<0.01$ \\
\hline & Prematurity & $0.99(0.77-1.29)$ & & $1.12(0.61-2.05)$ & & $0.95(0.61-1.46)$ & & $1.11(0.87-1.42)$ & \\
\hline & LBW & $1.40(1.07-1.83)$ & & $0.77(0.38-1.58)$ & & $1.75(1.28-2.39)$ & & $1.09(0.77-1.53)$ & \\
\hline & Asphyxia & $1.86(1.29-2.67)$ & & $3.02(1.83-4.96)$ & & $1.65(1.08-2.53)$ & & $1.92(1.33-2.76)$ & \\
\hline & Others & $1.59(1.15-2.19)$ & & $2.20(1.07-4.52)$ & & $1.53(1.10-2.11)$ & & $1.14(0.73-1.78)$ & \\
\hline \multirow{4}{*}{$\begin{array}{l}\text { No of } \\
\text { previous } \\
\text { deliveries }\end{array}$} & 0 & 1 & $<0.01$ & 1 & 0.31 & 1 & $<0.01$ & 1 & $<0.01$ \\
\hline & 1 & $0.83(0.74-0.93)$ & & $0.84(0.63-1.12)$ & & $0.84(0.74-0.95)$ & & $0.61(0.49-0.76)$ & \\
\hline & $2-3$ & $0.68(0.56-0.83)$ & & $0.79(0.55-1.13)$ & & $0.68(0.55-0.84)$ & & $0.50(0.40-0.62)$ & \\
\hline & $>3$ & $0.52(0.41-0.66)$ & & $0.72(0.50-1.05)$ & & $0.51(0.39-0.67)$ & & $0.35(0.26-0.45)$ & \\
\hline \multirow[t]{5}{*}{ Age (years) } & $<20$ & 1 & $<0.01$ & 1 & 0.02 & 1 & 0.01 & 1 & $<0.01$ \\
\hline & $20-24$ & $1.28(1.12-1.47)$ & & $1.75(1.25-2.44)$ & & $1.20(1.04-1.39)$ & & $1.37(1.12-1.67)$ & \\
\hline & $25-29$ & $1.56(1.27-1.93)$ & & $1.96(1.23-3.11)$ & & $1.49(1.16-1.92)$ & & $1.72(1.34-2.21)$ & \\
\hline & $30-34$ & $1.44(1.15-1.81)$ & & $1.66(0.99-2.78)$ & & $1.35(1.03-1.78)$ & & $1.98(1.50-2.61)$ & \\
\hline & $>34$ & $1.63(1.34-1.99)$ & & $1.62(1.00-2.64)$ & & $1.57(1.21-2.03)$ & & $2.24(1.75-2.85)$ & \\
\hline \multirow[t]{2}{*}{ Singletons } & Yes & 1 & $<0.01$ & 1 & 0.35 & 1 & 0.03 & 1 & 0.04 \\
\hline & No & $1.49(1.10-2.03)$ & & $1.31(0.74-2.33)$ & & $1.51(1.05-2.16)$ & & $1.58(1.03-2.43)$ & \\
\hline \multirow{3}{*}{$\begin{array}{l}\text { ARVs during } \\
\text { Pregnancy }\end{array}$} & None & N/A & & N/A & & N/A & & 1 & 0.83 \\
\hline & $<4 w k s$ & N/A & & N/A & & N/A & & $0.98(0.72-1.32)$ & \\
\hline & $\geq 4 \mathrm{wks}$ & N/A & & N/A & & N/A & & $0.96(0.81-1.12)$ & \\
\hline \multirow{2}{*}{$\begin{array}{l}\text { Facility } \\
\text { Ownership }\end{array}$} & Govt. & 1 & 0.90 & 1 & 0.57 & 1 & 0.36 & 1 & 0.77 \\
\hline & CHAM & $1.42(0.65-3.13)$ & & $1.27(0.55-2.94)$ & & $1.48(0.64-3.41)$ & & $1.09(0.62-1.90)$ & \\
\hline \multirow{2}{*}{$\begin{array}{l}\text { Facility } \\
\text { Location }\end{array}$} & Rural & 1 & 0.27 & 1 & 0.26 & 1 & 0.13 & 1 & 0.23 \\
\hline & Urban & $1.39(0.91-2.12)$ & & $1.19(0.88-1.62)$ & & $1.47(0.89-2.41)$ & & $1.16(0.91-1.46)$ & \\
\hline
\end{tabular}

OPL obstructed/prolonged labor, RU Ruptured Uterus, CHAM Christian Health Association of Malawi, LBW Low Birth Weight, aOR adjusted odds ratio, $A R V$ s antiretrovirals

strict CS indication setting by health care workers in recognition of the increased post-operative morbidity and mortality [28-30]. Clinicians may also be reluctant to perform ECS in HIV infected women due to a perceived risk of occupational HIV transmission [31]. Stigma and discrimination related to HIV infection may also play a role on health care workers' decision making
[32]. On the other hand, we observed that among HIV positive women the prevalence of CS and ECS was higher in those who had no or less than 4 weeks of exposure to ARVs (i.e. were at high MTCT risk) than in women with longer ARV drug usage in pregnancy. This suggests that high MTCT risk is being considered as an indication for CS in Malawian clinical practice. 
Table 4 Individual and facility-level factors associated with Elective Caesarean Section

\begin{tabular}{|c|c|c|c|c|c|c|c|c|c|}
\hline Item & & $\begin{array}{l}\text { aOR }(95 \% \mathrm{Cl}) \\
\text { All Women } \\
(55,443)\end{array}$ & $p$ & $\begin{array}{l}\text { aOR }(95 \% \mathrm{Cl}) \text { Women with } \\
\text { Unknown HIV status } \\
(n=4764)\end{array}$ & $p$ & $\begin{array}{l}\text { aOR }(95 \% \mathrm{Cl}) \\
\text { HIV- Women } \\
(n=29,747)\end{array}$ & $p$ & $\begin{array}{l}\text { aOR }(95 \% \mathrm{Cl}) \\
\mathrm{HIV}+\text { Women } \\
(\mathrm{n}=20,932)\end{array}$ & $p$ \\
\hline \multirow[t]{4}{*}{ Year of Admission } & 2010 & 1 & 0.03 & 1 & 0.01 & 1 & 0.02 & 1 & $<0.09$ \\
\hline & 2011 & $0.63(0.42-0.94)$ & & $0.47(0.29-0.76)$ & & $0.64(0.43-0.96)$ & & $0.84(0.55-1.30)$ & \\
\hline & 2012 & $0.55(0.34-0.88)$ & & $0.61(0.31-1.22)$ & & $0.53(0.33-0.86)$ & & $0.55(0.28-1.06)$ & \\
\hline & 2013 & $0.53(0.35-0.83)$ & & $0.40(0.21-0.78)$ & & $0.54(0.36-0.82)$ & & $0.68(0.39-1.19)$ & \\
\hline \multirow[t]{4}{*}{ No of deliveries } & 0 & 1 & $<0.01$ & 1 & 0.11 & 1 & $<0.01$ & 1 & $<0.01$ \\
\hline & 1 & $0.76(0.66-0.87)$ & & $0.74(0.57-0.96)$ & & $0.77(0.66-0.90)$ & & $0.59(0.46-0.75)$ & \\
\hline & $2-3$ & $0.65(0.51-0.82)$ & & $0.68(0.42-1.11)$ & & $0.65(0.51-0.83)$ & & $0.49(0.38-0.63)$ & \\
\hline & $>3$ & $0.46(0.34-0.63)$ & & $0.68(0.40-1.16)$ & & $0.45(0.31-0.65)$ & & $0.31(0.22-0.43)$ & \\
\hline \multirow[t]{5}{*}{ Age (years) } & $<20$ & 1 & $<0.01$ & 1 & $<0.01$ & 1 & $<0.01$ & 1 & $<0.01$ \\
\hline & $20-24$ & $1.38(1.18-1.61)$ & & $2.07(1.42-3.03)$ & & $1.29(1.09-1.53)$ & & $1.46(1.12-1.91)$ & \\
\hline & $25-29$ & $1.68(1.35-2.09)$ & & $2.11(1.21-3.67)$ & & $1.60(1.25-2.06)$ & & $1.86(1.33-2.60)$ & \\
\hline & $30-34$ & $1.46(1.10-1.92)$ & & $1.44(0.81-2.54)$ & & $1.37(1.00-1.88)$ & & $2.19(1.40-3.43)$ & \\
\hline & $>34$ & $1.68(1.24-2.27)$ & & $1.93(1.07-3.50)$ & & $1.58(1.08-2.32)$ & & $2.26(1.57-3.27)$ & \\
\hline \multirow[t]{2}{*}{ Singletons } & Yes & 1 & $<0.01$ & 1 & 0.13 & 1 & $<0.01$ & 1 & $<0.01$ \\
\hline & No & $1.98(1.55-2.54)$ & & $1.48(0.90-2.46)$ & & $2.06(1.54-2.77)$ & & $2.27(1.51-3.41)$ & \\
\hline \multirow[t]{3}{*}{ ARVs during Pregnancy } & None & N/A & & N/A & & N/A & & 1 & 0.04 \\
\hline & $<4 w k s$ & N/A & & N/A & & N/A & & $0.96(0.73-1.27)$ & \\
\hline & $\geq 4 w k s$ & N/A & & N/A & & N/A & & $0.81(0.68-0.96)$ & \\
\hline \multirow[t]{2}{*}{ Facility Ownership } & Government & 1 & 0.69 & 1 & 0.47 & 1 & 0.72 & 1 & 0.80 \\
\hline & CHAM & $1.63(0.69-3.83$ & & $1.19(0.74-1.91)$ & & $1.75(0.72-4.27)$ & & $1.08(0.59-1.99)$ & \\
\hline \multirow[t]{2}{*}{ Facility Location } & Rural & 1 & 0.03 & 1 & 0.01 & 1 & 0.03 & 1 & $<0.01$ \\
\hline & Urban & $1.95(1.06-3.56)$ & & $1.52(1.17-1.98)$ & & $2.02(1.05-3.86)$ & & $2.15(1.27-3.64)$ & \\
\hline
\end{tabular}

CHAM Christian Health Association of Malawi, $a O R$ adjusted odds ratio, ARVs antiretrovirals

We are not aware of other publications that assessed why women or health care workers opt for an ECS in subSaharan Africa. Most of the recent publications focused on the effect of early ART and Option B+ on PMTCT [33, 34]. A recent systematic review [35] assessed the risks and benefits of ECS in women with HIV. Of 36 studies included, only 3 were done in sub-Saharan Africa. Two of these studies described risk factors for MTCT [36, 37]. Unger et al. analyzed the use of CS over time, and assessed postpartum morbidity and mortality; but they did not discuss why the use of CS increased over time [38].

Our data showed that ECS was more frequent among women who delivered in urban than rural facilities. Several studies observed a higher prevalence of CS in urban settings and this was mostly driven by demands from mothers or by provider preferences [20,32,39] although it is also possible that the difference reflects the larger availability of surgery facilities in urban areas. The reason why this difference was only observed in analyses of ECS and not of all CS, may be due to the fact that demands from mothers and provider preferences are not formally accepted indications for CS in guidelines, thus are not reported in registers and delivery charts or due to the fact that knowledge about the potential use of CS for MTCT is higher in urban areas.

Some studies have shown a declining trend in CS upon wider availability of ARVs [40]. We also saw a reduction in CS and ECS deliveries after 2010, but remarkably not in HIV positive women, suggesting that other factors than increased ART coverage determined this [6].

The study has several strengths and limitations. It is based on a large dataset and includes health facilities in different regions in Malawi. Generalization of the results of this study needs to be considered with care because the data were from large health facilities only. Another limitation is that we used routinely collected medical records which may have affected data quality. The proportion of missing data from explanatory variables was below $5 \%$, except for infant complications (Table 1) and we believe it is unlikely that data were systematically missing for particular groups. In our study, the HIV status variable could be determined by HIV test results that became available before the onset of labour and immediately after delivery. In the latter case HIV status could not have impacted on a CS/ ECS decision. Because all HIV testing in Malawi is with rapid point-of-care tests that provide a result within 
30 min, most results would have been obtained before delivery, but we do not have data to document this. Further, we assumed that CS was elective when no obstetric indication was recorded, while there could simply be missing obstetric data. We limited this as much as possible by complimenting maternity register data with information from labour charts and theatre registers.

We did not have data on ART adherence or treatment interruptions. Health care workers may have considered CS more frequently in patients with poorer adherence because they know that suboptimal adherence impairs the preventive effect of PMTCT.

\section{Conclusions}

We have shown that patterns of CS in Malawi are largely determined by maternal and infant complications. Positive HIV status was inversely associated with CS delivery, possibly related to health care workers' perceived risk of occupational HIV transmission and the recognition of the known higher rate of post-operative complications. Our results leave open the possibility that CS is practiced as a means of PMTCT in Malawi as we found that ECS was significantly more common among women with a high risk of MTCT due to no or short exposure to ARVs in pregnancy in a setting with limited access to viral load testing. Further studies are needed to confirm our findings, including surveys among providers of CS services and qualitative research involving patients and practitioners about factors related ECS decisions including stigma and wealth.

\section{Additional file}

Additional file 1: Appendix 1. Word document describing the sampling strategy used in the study. (DOCX $24 \mathrm{~kb}$ )

\section{Abbreviations \\ aOR: adjusted odds ratio; ART: Antiretroviral therapy; ARVs: Antiretrovirals; CHAM: Christian Health Association of Malawi; Cl: Confidence interval; CS: Caesarean section; ECS: Elective caesarean section; IQR: Interquartile range; I- TECH: International Training and Education Center for Health; LBW: Low Birth Weight; MOH: Ministry of Health; MTCT: Mother to child transmission; OPL: Obstructed/prolonged labour; PEER: Partnerships for Enhanced Engagement in Research; PMTCT: Prevention of mother to child transmission}

\section{Acknowledgements}

We would like to acknowledge all the health facility staff and $\mathrm{MOH}$ and CHAM management who supported the data collection. Further we would like to thank all the data entry team who diligently entered the data management and staff of Baobab Health Trust and I-TECH Malawi for the support provided during this study.

\section{Funding}

This work was supported by the Bill and Melinda Gates Foundation (Global Health Grant OPP1090200), The United States Agency for International Development- National Institute of Health Initiative Partnerships for Enhanced Engagement in Research Health (PEER Health) grant AID OAA-A-11-0012. OK was supported by PROSPER Fellowship Grant (Grant 3233 B-150 934) and by a professorship grant from the Swiss National Science Foundation (\#PPOOP3_163878). The funding agencies played no role in the design of the study, the collection, analysis, and interpretation of data, and in writing the manuscript.

\section{Availability of data and materials}

The datasets analyzed during the current study are not publicly available since we did not get approval to provide the data online. The data are available from the corresponding author on request, conditional to approval by the Malawi Ministry of Health.

\section{Authors' contributions}

LT developed the study protocol, conducted the initial analysis and interpretation of data. OK, JJO, ADH and JE contributed in the data analysis and helped with the interpretation of the results. NP, MM, FC, KAP and KT contributed to the interpretation of the results. LT drafted the manuscript which was critically revised by OK, JJO, ADH, NP, MM, JE, FC, KAP and KT. FC, $\mathrm{MM}, \mathrm{ADH}$ and NP actively participated in data collection and management. All authors have read the final version of the manuscript and have given final approval of this version to be published.

Ethics approval and consent to participate

The study was granted ethical approval by the Malawi's National Health Sciences Research Committee (approval number 962) and Cantonal Ethics Committee of Bern, Switzerland. Because we only used routinely collected data, that were retrospectively extracted without personal identifiers, individual informed consent was waived by the two ethics boards.

Consent for publication

not applicable.

Competing interests

The authors declare that they have no competing interests.

\section{Publisher's Note}

Springer Nature remains neutral with regard to jurisdictional claims in published maps and institutional affiliations.

\section{Author details}

'Department of HIV and AIDS, Ministry of Health, Lilongwe, Malawi. ${ }^{2}$ Institute of Social and Preventive Medicine, University of Bern, Bern, Switzerland. ${ }^{3}$ International Training and Education Center for Health, P.O Box 30369, Lilongwe 3, Malawi. ${ }^{4}$ Dignitas International, Zomba, Malawi. ${ }^{5}$ Department of Medicine, College of Medicine, Blantyre, Malawi. ${ }^{6}$ The Baobab Health Trust, Lilongwe, Malawi. ${ }^{7}$ Department of Infectious Diseases, University Hospital Bern, Bern, Switzerland. ${ }^{8}$ Institute of Global Health, University of Geneva, Geneva, Switzerland. ${ }^{9}$ Institute of Mathematical Statistics and Actuarial Science, University of Bern, Bern, Switzerland.

Received: 7 March 2017 Accepted: 28 March 2018

Published online: 12 April 2018

References

1. UNAIDS. AIDSinfo 2014 [Available from: http://aidsinfo.unaids.org/. Accessed 18 June 2016

2. De Cock KM, Fowler MG, Mercier E, de Vincenzi I, Saba J, Hoff E, et al. Prevention of mother-to-child HIV transmission in resource-poor countries: translating research into policy and practice. JAMA. 2000;283(9):1175-82.

3. WHO. Global Update on HIV Treatment 2013.

4. Schouten EJ, Jahn A, Midiani D, Makombe SD, Mnthambala A, Chirwa Z, et al. Prevention of mother-to-child transmission of HIV and the health-related millennium development goals: time for a public health approach. Lancet. 2011;378(9787):282-4.

5. WHO. Consolidated guidelines on the use of antiretroviral drugs for treating and preventing HIV infection: what's new. 2015.

6 CDC. Impact of an innovative approach to prevent mother-to-child transmission of HIV-Malawi, July 2011-September 2012. MMWR Morb Mortal Wkly Rep. 2013;1(62):148-51.

7 Tenthani L, Haas AD, Egger M, Van Oosterhout JJ, Jahn A, Chimbwandira F, et al. Brief report: HIV testing among pregnant women who attend antenatal Care in Malawi. J Acquir Immune Defic Syndr. 2015;69(5):610-4.

8 Haas AD, Tenthani L, Msukwa MT, Tal K, Jahn A, Gadabu OJ, et al. Retention in care during the first 3 years of antiretroviral therapy for women in Malawi's option B+ programme: an observational cohort study. Lancet HIV. 2016;3(4):e175-82. 
9 Tenthani L, Haas AD, Tweya H, Jahn A, van Oosterhout JJ, Chimbwandira F, et al. Retention in care under universal antiretroviral therapy for HIV-infected pregnant and breastfeeding women ('Option B+') in Malawi. J Acquir Immune Defic Syndr. 2015;69(5):610-4.

10 Haas AD, Msukwa MT, Egger M, Tenthani L, Tweya H, Jahn A, et al. Adherence to antiretroviral therapy during and after pregnancy: cohort study on women receiving Care in Malawi's "option B+" programme. Clin. Infect Dis. 2016;63(9):1227-35.

11 Ministry of Health Malawi. Integrated HIV Program Report January-March 2016.

12 Brocklehurst P. Interventions for reducing the risk of mother-to-child transmission of HIV infection. Cochrane Database Syst Rev. 2002;(1). Art. No.: CD000102. https://doi.org/10.1002/14651858.CD000102.

13 European Mode of Delivery C. Elective caesarean-section versus vaginal delivery in prevention of vertical HIV-1 transmission: a randomised clinical trial. Lancet. 1999;353(9158):1035-9

14 Read JS, Newell MK. Efficacy and safety of cesarean delivery for prevention of mother-to-child transmission of HIV-1. Cochrane Database Syst Rev. 2005; (4). Art. No 4:CD005479. https://doi.org/10.1002/14651858.CD005479.

15 de Ruiter A, Taylor GP, Clayden P, Dhar J, Gandhi K, Gilleece Y, et al. British HIV association guidelines for the management of HIV infection in pregnant women 2012 (2014 interim review). HIV Med. 2014:15(Suppl 4):1-77.

16 Panel on Treatment of HIV-Infected Pregnant Women and Prevention of Perinatal Transmission. Recommendations for Use of Antiretroviral Drugs in Pregnant HIV-1-Infected Women for Maternal Health and Interventions to Reduce Perinatal HIV Transmission in the United States. Available at http:// aidsinfo.nih.gov/contentfiles/lvguidelines/PerinatalGL.pdf. Accessed 12 June 2016.

$17 \mathrm{WHO}$. Consolidated guidelines on the use of antiretroviral drugs for treating and preventing HIV infection: recommendations for a public health approach June 2013.' Available from: http://apps.who.int/iris/bitstream/ 10665/85321/1/9789241505727_eng.pdf?ua=1. Accessed 12 June 2016.

18 Fenton PM, Whitty CJ, Reynolds F. Caesarean section in Malawi: prospective study of early maternal and perinatal mortality. BMJ. 2003;327(7415):587.

19 WHO. Caesarean sections should only be performed when medically necessary. Switzerland: Geneva; 2015.

20 Buekens P, Curtis S, Alayon S. Demographic and health surveys: caesarean section rates in sub-Saharan Africa. BMJ. 2003:326(7381):136.

21 Macro. National Statistical Office. Calverton, Maryland: Malawi Demographic and Health Survey 2004; 2005.

22 Macro National Stastical Office. Malawi demographic and health survey 2010. Malawi, and Calverton, Maryland, USA: Zomba; 2011.

23 Chu K, Cortier H, Maldonado F, Mashant T, Ford N, Trelles M. Cesarean section rates and indications in sub-Saharan Africa: a multi-country study from Medecins sans Frontieres. PLoS One. 2012;7(9):e44484.

24 Carolan M. Maternal age $>/=45$ years and maternal and perinatal outcomes: a review of the evidence. Midwifery. 2013;29(5):479-89.

25 Carolan M, Frankowska D. Advanced maternal age and adverse perinatal outcome: a review of the evidence. Midwifery. 2011;27(6):793-801.

26 Cavazos-Rehg PA, Krauss MJ, Spitznagel EL, Bommarito K, Madden T, Olsen MA, et al. Maternal age and risk of labor and delivery complications. Matern Child Health J. 2015;19(6):1202-11.

27 Aebi-Popp K, Mulcahy F, Glass TR, Rudin C, Martinez de Tejada B, Bertisch B, et al. Missed opportunities among HIV-positive women to control viral replication during pregnancy and to have a vaginal delivery. J Acquir Immune Defic Syndr. 2013;64(1):58-65.

28 Ferrero S, Bentivoglio G. Post-operative complications after caesarean section in HIV-infected women. Arch Gynecol Obstet. 2003;268(4):268-73.

29 Kourtis AP, Ellington S, Pazol K, Flowers L, Haddad L, Jamieson DJ. Complications of cesarean deliveries among HIV-infected women in the United States. AIDS. 2014;28(17):2609-18.

30 Read JS, Tuomala R, Kpamegan E, Zorrilla C, Landesman S, Brown G, et al. Mode of delivery and postpartum morbidity among HIV-infected women: the women and infants transmission study. J Acquir Immune Defic Syndr. 2001;26(3):236-45.

31 Gounden YP, Moodley J. Exposure to human immunodeficiency virus among healthcare workers in South Africa. International journal of gynaecology and obstetrics: the official organ of the. Int J Gynaecol Obstet. 2000;69(3):265-70.

32 Neuman M, Alcock G, Azad K, Kuddus A, Osrin D, More NS, et al. Prevalence and determinants of caesarean section in private and public health facilities in underserved south Asian communities: cross-sectional analysis of data from Bangladesh, India and Nepal. BMJ Open. 2014;4(12):e005982.
33 Haas AD, van Oosterhout JJ, Tenthani L, Jahn A, Zwahlen M, Msukwa MT, et al. HIV transmission and retention in care among HIV-exposed children enrolled in Malawi's prevention of mother-to-child transmission programme. J Int AIDS Soc. 2017;20(1):21947.

34 Gourlay A, Birdthistle I, Mburu G, lorpenda K, Wringe A. Barriers and facilitating factors to the uptake of antiretroviral drugs for prevention of mother-to-child transmission of HIV in sub-Saharan Africa: a systematic review. J Int AIDS Soc. 2013;16:18588.

35 Kennedy CE, Yeh PT, Pandey S, Betran AP, Narasimhan M. Elective cesarean section for women living with HIV: a systematic review of risks and benefits. AIDS. 2017;31(11):1579-91.

36 Bobat R, Coovadia H, Coutsoudis A, Moodley D. Determinants of mother-tochild transmission of human immunodeficiency virus type 1 infection in a cohort from Durban, South Africa. Pediatr Infect Dis J. 1996;15(7):604-10.

37 Ilohl KK, Ilohl ON, Ikefunal AN, Ibeziakol NS, Ubesiell AC, Emodi IJ. Determinants of mother-to-child transmission of HIV despite PMTCT interventions in Enugu, Nigeria. S Afr J Child Health. 2015;9(2):49-52.

38 Unger JA, Richardson BA, Otieno PA, Farquhar C, Wamalwa D, John-Stewart GC. Mode of delivery and postpartum HIV-1 disease progression and mortality in a Kenyan cohort. BMC Pregnancy Childbirth. 2014;14:257.

39 Khanal V, Karkee R, Lee AH, Binns CW. Adverse obstetric symptoms and rural-urban difference in cesarean delivery in Rupandehi district, western Nepal: a cohort study. Reprod Health. 2016;13:17.

40 European Collaborative S, Boer K, England K, Godfried MH, Thorne C. Mode of delivery in HIV-infected pregnant women and prevention of mother-tochild transmission: changing practices in Western Europe. HIV Med. 2010; 11(6):368-78.

\section{Submit your next manuscript to BioMed Central and we will help you at every step:}

- We accept pre-submission inquiries

- Our selector tool helps you to find the most relevant journal

- We provide round the clock customer support

- Convenient online submission

- Thorough peer review

- Inclusion in PubMed and all major indexing services

- Maximum visibility for your research

Submit your manuscript at www.biomedcentral.com/submit
C) Biomed Central 